\title{
PROPUESTA PARA UNAAGENDA DE INVESTIGACIÓN DE LOS IMPACTOS DEL TURISMO EN LOS DESTINOS PATRIMONIO DE LA HUMANIDAD ${ }^{1}$
}

\author{
Juan Ignacio Pulido Fernández \\ Universidad de Jaén \\ jipulido@ujaen.es \\ Agustín Ruiz Lanuza \\ Universidad de Guanajuato \\ arlanuza@hotmail.com
}

\section{RESUMEN}

Este artículo tiene como objetivo integrar una agenda de investigación para el estudio de los impactos del turismo en los Sitios del Patrimonio Mundial de la UNESCO. Como antecedente, se ha realizado una revisión bibliográfica en Scopus de la que se han obtenido algunos de los posibles tópicos de investigación. Para la validación del interés científico de estos tópicos, se utiliza el método Delphi, con dos rondas. En la primera ronda participan 31 expertos y 21 en la segunda, logrando el consenso en veintinueve de las líneas de investigación propuestas inicialmente, y proponiendo siete nuevas líneas, de diversos campos del conocimiento, relacionados con el patrimonio y su uso turístico.

Palabras clave: agenda de investigación, turismo, Sitios del Patrimonio Mundial, Delphi.

\section{ABSTRACT}

This paper aims to integrate a research agenda for studying the impacts of tourism on World Heritage sites by UNESCO. As background, there has been a literature review in Scopus which were obtained some possible research topics. To validate the scientific interest of these topics,

Fecha de recepción: julio 2015.

Fecha de aceptación: abril 2016.

1 Este trabajo forma parte de una investigación financiada por el Consejo Nacional de Ciencia y Tecnología de México (CONACYT) del Programa de Estancias Posdoctorales al Extranjero. 
the Delphi method is used, with two rounds. In the first round involved 31 experts and 21 in the second, achieving consensus in twenty nine of the research lines originally proposed, and proposing seven new lines, from various fields of knowledge related to heritage and tourism.

Keywords: research agenda, tourism, World Heritage Sites, Delphi.

\section{INTRODUCCIÓN}

Hasta junio de 2015, se encuentran inscritos en la lista del Patrimonio Mundial de la UNESCO un total de 1.031 bienes. Adicionalmente, 1.629 bienes se encuentran en la lista tentativa a obtener el reconocimiento, muchas veces motivados, además de por la conservación, por la posibilidad del incremento de la actividad turística capaz de generar una serie de impactos positivos en el territorio. Algunos de estos destinos ya se encontraban posicionados turísticamente antes del reconocimiento de la UNESCO (París, Venecia, Guanajuato, etc.), otros destinos intentan pertenecer a esta lista y con ello obtener notoriedad turística (Ronda, Pátzcuaro, etc.). En ambos casos, dada la transversalidad del turismo, su estudio presenta una serie de aristas, que deben ser analizadas desde diversas disciplinas.

La excepcionalidad de los Sitios del Patrimonio Mundial (en adelante, SPM) puede deberse a uno o más de los diez criterios determinados por la UNESCO, cuya finalidad es estudiarlos, protegerlos, entenderlos y, en consecuencia, ponerlos en valor para el disfrute de propios y extraños. La visita a estos lugares, que son, en la mayoría de los casos, destinos turísticos únicos, genera una serie de retos y oportunidades de gestión que coinciden en todas las tipologías de destinos (García et al., 2011).

Esta problemática relación entre el turismo y los SPM ya fue planteada en los años ochenta del pasado siglo XX, en los que se consideraba al visitante como una amenaza potencial para el patrimonio (Coburn, 1984; Davis y Weiler, 1992). Batisse (1992) compara a la actividad turística desarrollada en los recursos patrimoniales, naturales o culturales, tangibles o intangibles, con la guerra y la contaminación. En los años noventa, la preocupación se centró en contener los flujos turísticos, evitando altas concentraciones (Wallace, 1993; Popp, 2001), mientras que en los 2000, Shafer e Inglis (2000) mencionan como un reto muy importante el conocer la capacidad de carga, que años más tarde evoluciona al concepto de capacidad de acogida (García et al., 2011). Es a partir de entonces cuando se valora la necesidad de ordenar la actividad turística, mediante planes específicos (Parlett et al., 1995), superando la idea de que la inclusión en la lista es un mero reconocimiento turístico (Wager, 1995) o una forma de ordenar al mundo (Lazzarotti, 2000). Esta planificación debe ser compartida (Hawes, 1996), sostenible, sobre todo implicando a la sociedad (Yuksel et al., 1999), con objeto de disminuir las insanas intenciones de los especuladores turísticos (Kirkpatrick, 2001).

Los beneficios más notorios del turismo en los SPM se obtienen cuando la visita está motivada, generalmente, por un interés por el patrimonio, en todas sus clasificaciones, lo que asegura la consolidación de estos destinos como las principales atracciones de turismo cultural (Mínguez, 2011), que experimentan un crecimiento de flujos turísticos muy importante, logrando la fidelidad del turismo hacia el patrimonio (Shen et al., 2014). Ello suele provocar una mayor conciencia patrimonial (Poria et al., 2013), aun cuando las actividades que realizan los turistas no sean necesariamente culturales (Brumann, 2014). 
El mantenimiento y cumplimiento de los compromisos devengados por el reconocimiento de la UNESCO también tiene un coste asociado, y los beneficios del turismo no están siempre garantizados (Wang y Zan, 2011), probablemente porque la marca SPM no está siendo potenciada o utilizada como se debiera. Incluso, muchos de estos destinos la excluyen de su promoción, o le dan un mayor protagonismo a las marcas nacionales (Beck, 2006).

El presente artículo tiene como principal objetivo el de establecer una agenda de investigación para el estudio del impacto de la actividad turística en los SPM. Para ello, una vez realizada una exhaustiva revisión bibliográfica, se utiliza la metodología Delphi, con la participación de expertos de diversas disciplinas y realidades territoriales, todos ellos vinculados directamente con el estudio, planificación y gestión de los SPM.

\section{MARCO TEÓRICO}

El impacto de la actividad turística en los SPM comienza a ser objeto de estudio desde hace más de tres décadas, desde diversas disciplinas y con diferentes enfoques. La mayor preocupación, en general, es la sostenibilidad de los recursos culturales y naturales que pertenecen a la Humanidad. Gran parte de los estudios consideran tradicionalmente al turismo como una actividad nociva para el patrimonio, destacan problemas propios del turismo, como son la estacionalidad (Jeon et al., 2014), la corta estancia (Roque et al., 2012), la escasa diversificación de mercados (Piédrola et al., 2012), las amenazas y oportunidades de los mercados asiáticos, principalmente, al mundo occidental (Winter, 2007), las consecuencias sociales que se generan, como la desigualdad social y marginación (Tucker y Emge, 2010). En suma, conflictos que presentan una serie de incidencias sociales y económicas que no han sido lo suficientemente estudiadas (Yang y Wall, 2014).

Una de las principales preocupaciones de los investigadores se orienta hacia la gestión del destino, tópico en el que se afrontan retos prácticamente desde sus inicios, muchos de los cuales aún no han sido superados y siguen siendo preocupaciones a las que se suman otras más recientes, inclinándose hacia la gestión de la política pública. Así, se observa de manera general que no se está gestionando de forma sostenible, según lo planteado por la UNESCO (Jureniene y Radzevicius, 2014). De hecho, la mayor parte de las publicaciones sobre esta cuestión plantean como el mejor camino de solución la gobernanza (Fatin et al., 2014; May, 2014). Por otro lado, Su y Lin (2014) y Wu et al. (2014) consideran que los visitantes internacionales a estos sitios obtienen un bien complementario, que no necesariamente por sí mismo tiene que tener un coste, que incrementa su experiencia.

Desde la escala territorial, la preocupación de investigación se encuentra en los límites de protección, en los que se observa una marcada atención y mejora únicamente en los centros históricos de las ciudades, o lo puramente turístico y, por tanto, son más vigilados (Little, 2014), priorizando, a su vez, la inversión en infraestructuras públicas de cara al turismo, lo que conduce a impactos ambientales negativos en el resto de los espacios (Hennesy y Mccleary, 2011). Los grandes retos que mencionan los diversos autores son el estudio de la capacidad de acogida, mediante enfoques científicos, de acuerdo a metodologías adaptativas planteadas por diversos organismos internacionales, en los principales recursos culturales (García y De la Calle, 2012; Larson y Poudayl, 2012). Gestionar los flujos turísticos es una cuestión clave (Duval y Gauchon, 2007; Fernández Carrasco, 2007), ya que permitirá afrontar la saturación turística y la 
estacionalidad (Zhang et al., 2014). Por otro lado, Su y Lin (2014) y Su y Wall (2013) afirman que, de manera general, se estudia más su estructura de gestión y la falta de recursos financieros para hacer frente a los impactos sobre el patrimonio. Concluyen que existe la necesidad de articular más estudios de casos, con mayor detalle, con conceptos claros y marcos teóricos en una diversidad de sitios, para conducir a la comprensión de la relación entre turismo y patrimonio.

Gran parte del análisis se realiza mediante estudios de casos, con una visión más positiva. De especial interés es el estudio realizado por Hawkins et al. (2009), en el que evalúan treinta y tres SPM utilizando los informes del Centro de Destinos Sostenibles del National Geographic, en un período de 2003 a 2006, mediante una encuesta con metodología Delphi a otros expertos y stakeholders de los propios destinos, en los que observan que más del $27 \%$ de estos sitios ha mejorado turísticamente de forma considerable.

En el análisis de Lozano y Gutiérrez (2011) esta visión es compartida y adicionan que son sitios que disfrutan de una ventaja competitiva respecto a oferta y empleo, y coinciden en que el número de SPM contenidos en cada país le da un plus a éste de cara al turismo internacional (Yang et al., 2010; Poira et al., 2011), como lo demuestran Zhang et al. (2014) en estudios realizados con una muestra de sesenta y seis países.

La medición de los impactos del turismo en los SPM se sugiere necesaria, así como también avanzar en metodologías capaces de evaluar los impactos económicos de manera sistemática (Viu et al., 2008; Su y Wall, 2014), ya que, por un lado, los actores públicos y privados invierten en el mantenimiento y conservación de monumentos y, por otro, las personas gastan para visitarlos (Todt et al., 2008). E, incluso, ir más allá, dado que, por ejemplo, en Corea, utilizando el método de valoración contingente, resulta mayor el valor económico del patrimonio que sus beneficios monetarios (Kim et al., 2007). Es necesario generar mecanismos que permitan evaluar la experiencia de los visitantes, superando los estudios clásicos relacionados con el movimiento y gasto de los flujos turísticos (Daengbuppha et al., 2006).

Hace falta realizar estudios que tengan en cuenta a la sociedad, como los llevados a cabo en la Bahía de Ha Long, en Vietnam, en los que la sociedad valora positivamente la actividad turística, aun cuando son cuestionables los resultados generados por el turismo (Long, 2012). En algunos SPM, como Safranbolu, en Turquía (Turker, 2013), la sociedad receptora ha observado en un período de cinco años un cambio importante, traducido en mejor empleo, oportunidades de negocio y desarrollo comunitario. Es importante evitar lo que sucede en casos como la comunidad Naxi, en Lijiang, en la que sus casas quedaron convertidas en alojamientos turísticos y tiendas de recuerdos, es decir, ha caído en una mercantilización turística (Su y Teo, 2008).

En concreto, el estudio del turismo en los SPM, dada su transversalidad, ofrece diversos campos de investigación, con la singularidad de que siempre debe tomarse en cuenta el patrimonio como la base del producto turístico, lo que plantea un esfuerzo adicional.

\section{METODOLOGÍA}

Este trabajo tiene como antecedente una investigación que se aproxima rigurosamente al estado de la cuestión en relación a los impactos del turismo sobre los destinos incluidos en la lista del Patrimonio Mundial de la UNESCO, realizada mediante la revisión de la literatura localizada en Scopus de Elsevier (Ruiz y Pulido, 2015). 
En esta revisión se encontraron 961 artículos que contenían las palabras "patrimonio mundial" y "turismo". Estos artículos fueron analizados y sintetizados bajo el método de "análisis de contenido" (Pokharel y Mutha, 2009). De ahí, se seleccionaron 178 aportaciones, publicadas entre 1981 y 2015 , que evidencian algunas incidencias del turismo en SPM, no importando si estas incidencias son consecuencia del reconocimiento por parte de la UNESCO. Una parte importante de estos artículos están publicados en revistas de contenidos turísticos provenientes, principalmente, de universidades de Estados Unidos, Reino Unido, China, Australia e Italia. En su mayoría, estudiados desde las Ciencias Sociales, la Administración y Negocios, así como el Medioambiente. En la Tabla 1 se incluyen las 23 revistas con un mayor número de publicaciones.

Tabla 1

PUBLICACIONES REGISTRADAS EN SCOPUS, CON MAYOR NÚMERO DE CONTRIBUCIONES ANALIZADAS

\begin{tabular}{|c|c|c|c|c|c|c|c|c|c|c|c|}
\hline Nombre & $\mathbf{N}^{\circ}$ & Nombre & $\mathrm{N}^{0}$ & Nombre & $\mathrm{N}^{a}$ & Nombre & $\mathbf{N}^{0}$ & Nombre & $\mathbf{N}^{\circ}$ & Nombre & $\mathrm{N}^{a}$ \\
\hline $\begin{array}{c}\text { Journal of } \\
\text { Sustainable Tourism }\end{array}$ & 10 & \begin{tabular}{|c|}
$\begin{array}{c}\text { Asia Pacific } \\
\text { Journal of } \\
\text { Tourism Research }\end{array}$ \\
\end{tabular} & 4 & $\begin{array}{l}\text { Advanced } \\
\text { Materials } \\
\text { Research }\end{array}$ & 2 & Anatolia & 2 & Facilities & 2 & Milli Folklor & 2 \\
\hline $\begin{array}{c}\text { Tourism } \\
\text { Management }\end{array}$ & 10 & $\begin{array}{c}\text { Annals of } \\
\text { Tourism Research }\end{array}$ & 4 & $\begin{array}{c}\text { International } \\
\text { Journal of } \\
\text { Tourism Research }\end{array}$ & 2 & $\begin{array}{l}\text { International } \\
\text { Journal of } \\
\text { Services } \\
\text { Technology and } \\
\text { Management }\end{array}$ & 2 & $\begin{array}{l}\text { Island Studies } \\
\text { Journal }\end{array}$ & 2 & $\begin{array}{l}\text { Wit Transactions } \\
\text { on the Built } \\
\text { Environment }\end{array}$ & 2 \\
\hline $\begin{array}{c}\text { International } \\
\text { Journal of Heritage } \\
\text { Studies }\end{array}$ & 8 & $\begin{array}{l}\text { Wit Transactions } \\
\text { on Ecology and } \\
\text { the Environment }\end{array}$ & 4 & $\begin{array}{l}\text { Critical Asian } \\
\text { Studies }\end{array}$ & 2 & $\begin{array}{l}\text { Annales De } \\
\text { Geographie }\end{array}$ & 2 & $\begin{array}{c}\text { Lecture Notes in } \\
\text { Computer Science }\end{array}$ & 2 & Tourism & 2 \\
\hline $\begin{array}{l}\text { Current Issues in } \\
\text { Tourism }\end{array}$ & 4 & $\begin{array}{c}\text { Journal of Travel } \\
\text { Research }\end{array}$ & 2 & $\begin{array}{c}\text { Anales De } \\
\text { Geografia De La } \\
\text { Universidad } \\
\text { Complutense }\end{array}$ & 2 & $\begin{array}{l}\text { International } \\
\text { Journal of } \\
\text { Cultural Policy }\end{array}$ & 2 & Milli Folklor & 1 & Otros & 102 \\
\hline
\end{tabular}

Fuente: Ruiz y Pulido (2015: 1249).

Una vez seleccionados, se clasificaron siguiendo la propuesta de Webster y Watson (2002) y se agruparon en ocho tópicos clave: la gestión, la sostenibilidad, los flujos turísticos, la gestión territorial, el marketing, las incidencias sociales, la gestión patrimonial y los impactos económicos. Estos tópicos fueron obtenidos de los contenidos de las publicaciones.

Como se recoge en la Tabla 2, y como consecuencia del análisis de contenido de estas 178 aportaciones, se diferenciaron tres grandes grupos de publicaciones. En el primero se incluyen las aportaciones que abordan aspectos generales y conceptuales del turismo y los SPM. El segundo grupo se compone de los estudios realizados en destinos consolidados, y en un tercero se han agrupado los estudios de destinos que se encuentran en consolidación y que ven en el turismo un elemento de desarrollo endógeno (Beeson y Doganer, 2013). A su vez, se diferenciaron otros tres grupos, el primero muestra las experiencias que, a juicio de los autores, han sido perjudiciales o no responden a los objetivos planteados en el texto de la Convención de 1972 y sus posteriores directrices prácticas, que se denominaron "negativas". El segundo grupo muestra las experiencias en las que se observó una mejora sostenible del turismo, y que se han denominado "positivas" y, en un tercer grupo, se incluyen las aportaciones que contienen propuestas de cara a que el turismo genere beneficios sostenibles, a las que se han denominado "propuestas". 


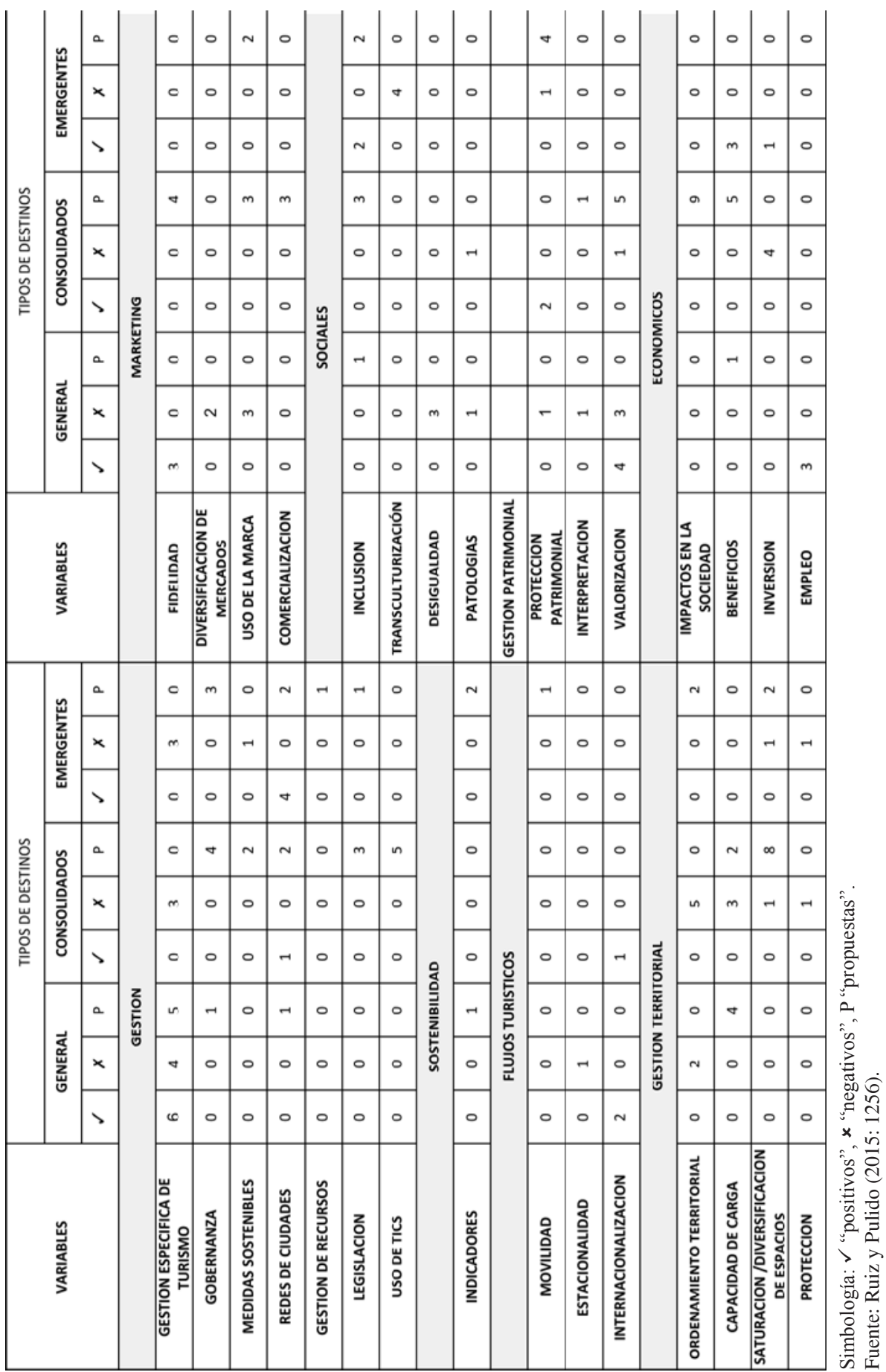


En principio, los temas en los que se encontró un menor número de publicaciones son aquellos en los que se puede identificar una línea de investigación prioritaria. Para el caso del presente trabajo, son éstas las líneas que se han propuesto discutir a los expertos, pero sin un carácter excluyente, pues se ha facilitado durante el proceso la opción de que ellos pudieran identificar nuevas líneas de investigación.

Para cumplir el objetivo planteado, se ha utilizado el método Delphi, que en el ámbito del turismo ha sido utilizado desde hace, al menos, cuarenta años, sobre todo para la predicción de eventos, pronóstico de la evolución de la demanda turística y las tendencias del mercado.

La mayoría de estos estudios suelen quedar resueltos en una segunda ronda, e integrados, en un $65 \%$, por grupos menores de 30 personas (Vera y Song, 2014), aun cuando, bajo esta metodología, el tamaño del grupo de expertos no determina la calidad de los resultados (Garrod y Fyall, 2000). En este caso, como se recoge en la Tabla 3, el estudio consiste en dos rondas. En la primera participaron 31 expertos del tema. En la segunda ronda participaron 21 expertos, es decir se cuenta con una tasa de respuesta del $67,74 \%$ respecto a la primera ronda.

Tabla 3

FICHA TÉCNICA DEL MÉTODO DELPHI

\begin{tabular}{|c|l|}
\hline $\begin{array}{c}\text { Ámbito de } \\
\text { estudio }\end{array}$ & $\begin{array}{l}\text { Expertos internacionales en estudio, planificación y gestión de los Sitios } \\
\text { Patrimonio de la Humanidad, de los sectores académico, público y privado, } \\
\text { conocedores de los impactos generados por el turismo }\end{array}$ \\
\hline $\begin{array}{c}\text { Tamaño de la } \\
\text { muestra }\end{array}$ & $\begin{array}{l}31 \text { cuestionarios en la primera ronda } \\
21 \text { cuestionarios en la segunda ronda }\end{array}$ \\
\hline $\begin{array}{c}\text { Fecha del } \\
\text { trabajo empírico }\end{array}$ & $\begin{array}{l}\text { Inicio: } 1 \text { de junio de } 2015 \\
\text { Fin: } 30 \text { de julio de } 2015\end{array}$ \\
\hline Tipo de estudio & Delphi \\
\hline
\end{tabular}

Fuente: elaboración propia.

Lo que resulta muy importante en esta metodología es la selección de expertos. Kollwitz (2011) señaló que uno de los problemas asociados con la técnica Delphi es la identificación de un grupo apropiado de expertos, ya que representan el equilibrio deseado de opiniones, filosofías y la experiencia (Wheeller et al., 1990). Para este caso, se especifica el perfil de los participantes, no su nombre y adscripción, dado que su anonimato es fundamental (Rodríguez y Pulido, 2010).

El panel se integró con participantes de diferentes lugares de la geografía mundial: Argentina, Austria, Brasil, España, Estados Unidos de Norte América, Malasia y México. El $71 \%$ son académicos, miembros de los grupos de investigación más consolidados en el tema, el $13 \%$ son empresarios del sector turístico y el 16\% son gestores públicos, con representatividad de la UNESCO, el ICOMOS, la OCPM, asociaciones de ciudades patrimoniales, entre otros. El grado de conocimiento de los expertos participantes es muy alto, ya que el $23 \%$ ha trabajado más de 10 años en el tema, el $45 \%$ de 9 a 7 años, el 19\% de 4 a 7 años y el $13 \%$ de 1 a 3 años. El 100\% de los participantes conoce el proceso de integración de los expedientes de los SPM para la candidatura ante la UNESCO. El 13\% ha participado en la integración de algún expediente y el $13 \%$ ha participado en estudios posteriores. 
El estudio se resolvió en dos rondas. La primera ronda se integró con un cuestionario que se dividió en nueve apartados. El primero de ellos contiene tres preguntas para determinar el perfil del grupo, tres preguntas introductorias al tema y dos más que se integraron al estudio Delphi. Las restantes ocho secciones corresponden a los tópicos anteriormente señalados, con cinco preguntas en cada uno de ellos. Adicionalmente, se incluyó un espacio para que los expertos pudieran proponer alguna línea de investigación no contemplada.

La segunda respuesta de los participantes replica a la primera, exceptuando la información del perfil y las preguntas introductorias, además de que se incluyeron las líneas de investigación propuestas por los participantes en la primera ronda. Para obtener una mayor participación en el momento de presentar los primeros resultados, fue necesario integrar un cuestionario individual para cada participante en el que se mostraba el resultado de la primera ronda y la posición de su respuesta. En ambas rondas fue utilizada la aplicación de la herramienta "Google forms", lo que, sin duda, simplificó el envío de ambos cuestionarios y el tratamiento de la información, permitiendo que se resolviera en aproximadamente dos meses.

El tratamiento de los resultados se realizó utilizando como medida de concentración la mediana (Q2), mientras que la medida de dispersión utilizada ha sido el recorrido intercuartílico (RI=Q3-Q1). Algunos estudios han sugerido que un acuerdo superior al $60 \%$ de un panel se puede ver como el consenso del grupo (Hill y Fowles, 1975), mientras que otros han argumentado que el rango intercuartílico debe ser no más de $10 \%$ mayor o menor que la mediana (Frechtling, 2001).

Siguiendo las recomendaciones de Donohoe y Needham (2009), en este trabajo, por una parte, se considerará que se ha alcanzado un gran consenso cuando el $60 \%$ de los expertos, o más, haya optado por el valor de la mediana; bastante consenso cuando entre el $30 \%$ y el $59,99 \%$ de los expertos haya elegido el valor de la mediana; y un escaso consenso cuando menos del $30 \%$ de los expertos consultados haya elegido como respuesta el valor de la mediana. Y, por otra parte, se estimará que existe mucho consenso cuando el recorrido intercuartílico no supere el 0,7 (que sería el 10\% de los 7 puntos de la escala Likert utilizada), un considerable consenso cuando el recorrido intercuartílico alcance valores que oscilan entre 0,7 y 1,5 , y que no existe consenso suficiente cuando el valor del recorrido intercuartílico supere el 1,5.

\section{RESULTADOS Y DISCUSIÓN}

Los resultados del presente estudio se pueden ordenar en tres grupos. El primero tiene en cuenta las preguntas que se realizaron por primera vez, que no fueron parte de la metodología Delphi. El segundo considera las cuestiones que se evaluaron bajo la metodología Delphi y que prácticamente son las que contrastan entre el estudio previo de revisión de literatura y los expertos. Y un tercero, en el que se adicionan siete líneas de investigación propuestas por los expertos en la primera ronda del estudio y consensuadas en la segunda ronda.

Para el primer grupo, se realizaron tres preguntas de "encuadre" o introductorias, que no formaron parte del estudio Delphi. La primera es en relación a si los encuestados consideran que las propuestas de investigación deben ser aplicadas a los destinos en general o diferenciadas por el grado de consolidación de los mismos. Como se recoge en la Figura 1, el 78\% de los expertos afirmó que deberían ser diferenciadas, mientras que el 22\% afirmó que pue- 
den ser propuestas indistintas. No obstante, a los que opinaron que debería ser diferenciado, se les cuestionó en qué tópicos deberían serlo y los resultados apuntan a que las líneas de investigación deben ser planteadas con carácter general, pero con diferentes enfoques, de acuerdo a la realidad territorial. En esta pregunta hay cuatro aspectos que, según los expertos, deben ser estudiados especialmente en destinos consolidados, que son:

- Flujos turísticos. Coincidiendo con Jeon et al. (2014), quienes afirman que, aun cuando la estacionalidad en los SPM no llega a ser tan acusada como en los destinos de playa, también existe temporada baja, lo que, sumado a la corta estancia (menor a dos noches de alojamiento) característica de la mayoría de los destinos urbanos e históricos (Roque et al, 2012), provoca niveles de ocupación muy bajos, generando tarifas muy altas y concentraciones de flujos turísticos, lo que altera la experiencia del visitante.

- Gestión territorial. Reconociendo el espacio patrimonial como un anhelado destino alternativo principal o secundario de aquellos destinos más visitados, donde su atractivo radica en la originalidad, por lo que se debe evaluar el nivel de tercerización, gentrificación y despoblamiento de los centros históricos que, como afirman Yamamura (2006) y Sichenze (2006), reducen su contenido inmaterial y, por tanto, la experiencia de quienes la visitan.

- Gestión cultural. Preparando a los SPM como destinos culturales, adaptados a las necesidades de los visitantes y a las nuevas tendencias, como es el caso de las industrias creativas y el turismo, es decir la cultura puesta al servicio del turismo, como mencionan Aas, Ladkin y Fletcher (2005).

- Impactos sociales, que poco han sido estudiados en los destinos, tal como señalan Su y Wall (2014), quienes afirman que debe incluirse, además, a la comunidad de acogida en lugares cercanos, pues, en ocasiones, éstos se pueden ver beneficiados o afectados por los visitantes.

La siguiente pregunta buscaba la opinión sobre el cumplimiento de los postulados internacionales que intentan regular la actividad turística de forma sostenible en los SPM. El 37\% respondió que se cumplen algunos, el $40 \%$ opinó que se cumplen medianamente, el $23 \%$ afirmaron que no se cumplen y no hubo respuestas para decir que se cumplen totalmente, lo que justifica la medida emitida por la UNESCO desde 2011, en la que solicita a los destinos que conformen y cumplan con un plan específico de gestión turística, que incluya la valorización patrimonial reconocida. En este sentido, Dong (2010) y Bell (2013) afirman que la interpretación se debe centrar en los valores excepcionales que justifican su inclusión en la lista del patrimonio mundial, incluso, pueden diversificar el producto, evitando la idea tan sintetizada del destino descrita por Kavoura y Bitsani (2013).

La tercera pregunta de este primer grupo está relacionada con el conocimiento de los involucrados acerca de los criterios de excepcionalidad reconocidos por la UNESCO en cada destino. Las respuestas, en un $43 \%$, afirman que son totalmente desconocidos, un $27 \%$ medianamente conocidos, un $17 \%$ desconocidos y solamente un $13 \%$ opinan que sí son conocidos. Mouriño (2013) ya explicaba, mediante un modelo de regresión ordinal realizado en Sintra, que la experiencia patrimonial es determinada por el grado de conocimientos previos a la visita cultural, debido a la falta de interpretación en el sitio. 


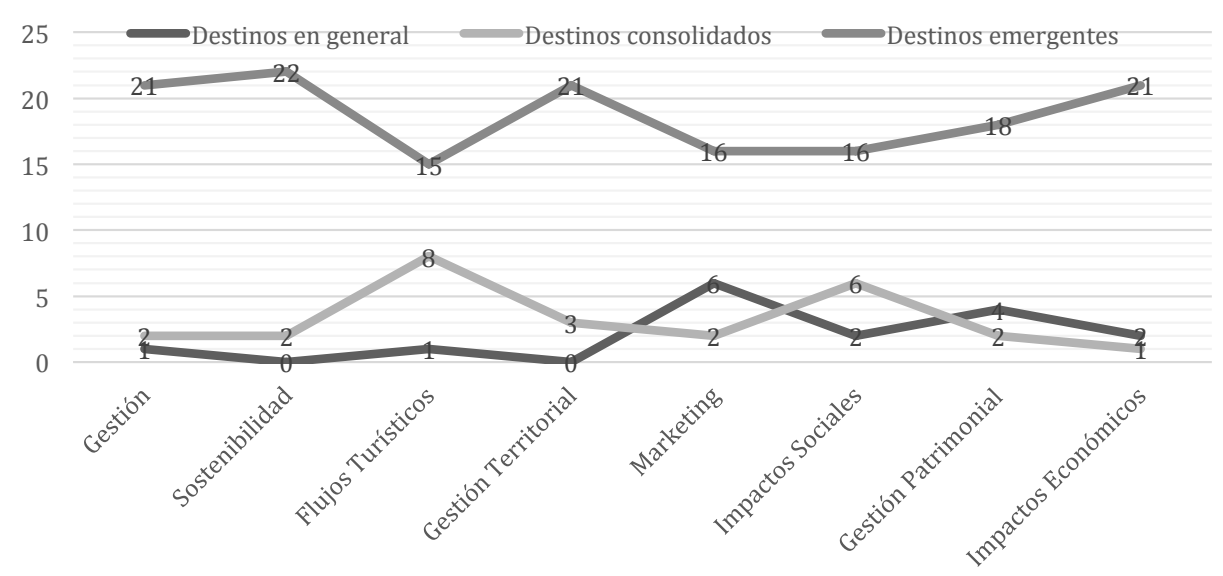

Fuente: elaboración propia.

Los resultados de las cuestiones bajo la metodología Delphi se obtienen después de conocer el recorrido intercuartílico de cada una de las rondas, como se estableció en la metodología, considerándose que el consenso se encuentra cuando se observan valores iguales o menores a 1, que es, también, el momento que nos indica la necesidad de establecer más rondas a los participantes, siendo prácticamente imposible conseguir un 100\% de participación, dado que, a medida que se incrementan las rondas, se disminuye el interés de parte de los participantes (Pulido, 2007). Los resultados que se mencionan a continuación se recogen en la Tabla 4, en la que se puede observar que, de forma general, en la segunda ronda se alcanza el consenso en la mayoría de los aspectos considerados.

La primera pregunta del Delphi es acerca de la importancia del reconocimiento de la UNESCO en el incremento de flujos turísticos en los SPM. El consenso se alcanza en la segunda ronda, resultando "importante", coincidiendo con los estudios de Hawkins et al. (2009), en los que se constata un incremento de visitantes a los SPM. Es evidente que el incremento de visitantes va a depender del estadio evolutivo en el que se encuentre el destino, ya que, en el caso de los consolidados, como comenta Palau (2013), ya atraían a un número importante de visitantes antes de ser reconocidos por la UNESCO y, en el caso de los destinos emergentes, la preocupación se centra en conquistar nuevos viajeros, con fines de desarrollo local, como afirman Wu et al. (2012) y Pearce (2014), por lo que, finalmente, se observa que el reconocimiento UNESCO contribuye a atraer visitantes y a mantener los niveles de ocupación del SPM.

La pregunta dos plantea la contribución del turismo a la preservación del patrimonio. La respuesta en la primera ronda se situaba mayoritariamente en la opción de que no afecta ni beneficia. Sin embargo, en la segunda se alcanza el consenso para la opción de que en muy pocas veces el turismo contribuye a la preservación del patrimonio. Coincide con la tradición científica para esta cuestión, que ha situado al turismo como una seria amenaza para el patrimonio (Couburn, 1984). En esta misma línea se expresan Davis y Weiler 
(1992), siguiendo la postura radical de Batisse (1992), que compara la actividad turística con la guerra. Es necesario incrementar estudios en los que se refleje una buena experiencia o contribución del turismo al patrimonio.

Una vez analizadas las preguntas orientativas y generales del tema, que evidencian una importante complejidad para su estudio, se procede a presentar los resultados para cada uno de los ocho tópicos que contienen las futuras líneas de investigación, obtenidas de la revisión bibliográfica y mencionadas en la Tabla 2.

El primer tópico, relacionado con la gestión de los destinos, es el que ofrece un mayor número de posibles líneas de investigación. Se someten cinco a la opinión de los expertos, alcanzándose un consenso total en tres de ellas, y un alto grado de consenso en las otras dos. La primera es investigar acerca de los instrumentos de planificación específica de turismo, pues, como comentan Baker y Cameron (2008), se debe contar con estrategias y planes de turismo más o menos homologados, aunque, como argumenta Jimura (2011), cuidando su adaptabilidad a cada realidad territorial y teniendo en cuenta un número importante de factores, que van desde la propia legislación hasta la formación profesional de sus responsables. La siguiente línea es investigar la inclusión de las TICs para convertirlos en destinos inteligentes, desde su propia gestión, en red, con otros SPM, ya que, incluso, los horarios son conflictivos cuando se trabaja en red a escala mundial. La tercera línea es investigar acerca de los instrumentos normativos en materia de turismo y de conservación patrimonial, dado que son bienes que han sido declarados Patrimonio de la Humanidad.

El siguiente tópico es el de la sostenibilidad. En la segunda ronda se alcanza el consenso respecto a la necesidad de investigación solo sobre dos líneas, que son: la aplicación de los recursos económicos generados por el turismo respecto al patrimonio, siendo una relación ya descrita por Kim, Wong y Cho (2007), en la que debe primar la consecución de las oportunidades económicas explicadas por Xu y Dai (2012). Y la otra línea de este tópico es el estudio de impacto ambiental dentro de los límites de protección del SPM, pero también en sus alrededores, evitando los impactos negativos, tal como mencionan Hennesy y Mccleary (2011). Con un menor nivel de consenso, aunque igualmente interesante, es la tercera línea de investigación propuesta relacionada con la necesidad de establecer indicadores de sostenibilidad turística para los SPM.

El tercer tópico es la que lleva por título flujos turísticos. No es hasta la segunda participación de expertos, cuando los expertos llegan al consenso total sobre las cuatro líneas que deben investigarse prioritariamente. Por un lado, la movilidad de visitantes dentro y fuera de los SPM, coincidiendo con lo que planteaban Bernier y Sutton (2012), dado que, en ocasiones, los SPM pueden ser el atractivo principal o, en otros casos y más frecuentemente, son atractivos complementarios, y es entonces cuando se deben de establecer nuevas rutas e itinerarios. La siguiente línea de investigación debe considerar los niveles de estacionalidad, tal como sugieren Joen y Kang (2014), mediante estudios sobre cómo afecta la estacionalidad a los residentes, por ejemplo, a los niveles de empleo, en temporadas de poca afluencia, que suelen bajar de manera importante; o en temporadas muy altas se altera el orden de los anfitriones, siendo el flujo turístico indeseable, perjudicando también la experiencia de los visitantes. La siguiente línea de investigación son las motivaciones de los visitantes desde su origen, en la que debe reconocerse la imagen que propicia el marketing, relacionándolo con las actividades que se llevan a cabo en el destino. 
La cuarta línea propuesta de investigación está relacionada con la experiencia y comportamiento de los visitantes en los SPM, que, si bien son destinos en los que, como establece Okech (2010), se practica el turismo cultural, siguiendo a Bruman (2014), la mayoría de las actividades de los visitantes no son culturales, con lo que, en ocasiones, se gestionan los destinos con un alto contenido cultural, hasta el punto de que no es posible visitarlos, y menos comprenderlos, en todo su esplendor.

El cuarto tópico, denominado análisis territorial, se compone de cinco ítems en los que se alcanza un consenso total. Por consiguiente, todas se consideran como líneas de investigación de interés. Una de ellas está relacionada con el estudio de la capacidad de carga turística de los SPM, tal como sugieren, entre otros, García y De la Calle (2012), dado que su análisis proporciona una serie de elementos que pueden determinar la base de la planificación, incluso en términos de marketing. Otra línea es la que atiende a la saturación y diversificación de espacios turísticos en los SPM, intentando mitigar los efectos negativos que provocan las altas concentraciones de visitantes. La siguiente línea prioritaria de investigación son los niveles de protección del patrimonio edificado, dado que no existen criterios homologados a nivel internacional sobre los que deben de conservarse para la Humanidad. Una línea más es la investigación de los Planes de Ordenamiento Territorial, específicamente en materia turística, dado que es un punto de partida en la planificación territorial de los SPM. Finalmente, otra línea más es observar el nivel de tercerización y gentrificación de sus centros históricos.

Para el quinto tópico, relacionado con temas de marketing, en la segunda oportunidad de respuesta los expertos acercan sus posturas respecto a la importancia de las cuatro líneas propuestas. La primera es la fidelidad de los visitantes, coincidiendo con los estudios de Ryan y Silvanato (2009), o el trabajo de Shen, Guo y Wu (2014), realizado en Nanjing, en el que observa la alta repetición de la visita con fines extraculturales. Otra línea es la diversificación de mercados, como el ejemplo que establecen Piédrola, Artacho y Villaseca (2012), cuando estudian el segmento enfocado al turismo idiomático, especializando una parte de la oferta del destino. Una línea más es evaluar la marca UNESCO, en un sentido turístico, que sea reconocible para los posibles visitantes, homologada y centrada en los valores de excepcionalidad. La última línea de este tópico plantea investigar los modelos de comercialización turística en los SPM, desde la propia promoción hasta la influencia de turoperadores.

El tópico de impactos sociales muestra cuatro líneas que fueron consensuadas en la segunda ronda por los expertos como muy importantes a investigar. La primera es el estudio de la inclusión de la sociedad en las actividades turísticas, para que, como argumentan Kavoura y Bistani (2013), exista una verdadera interpretación patrimonial de acuerdo a los saberes de la población local, provocando lo que Berliner (2012) menciona como fuerza impulsora en la protección y puesta en valor del patrimonio. Una línea más es analizar el nivel de transculturización provocado por los visitantes, sobre todo en los destinos emergentes, en los que se conserva una autenticidad, como sucede en cuestiones religiosas, abordadas por Cohen (2014), ya que lo que para los visitantes puede ser atractivo, para la comunidad anfitriona son momentos íntimos con un fuerte componente espiritual. En este sentido, es necesario discernir entre lo turístico y lo que debe estar reservado para no importunar a la sociedad de acogida. La siguiente línea es la desigualdad social causada por el turismo, consecuencia de que los beneficios del turismo no se reparten de manera equitativa para todos los habitantes del destino, sobre todo para el personal de contacto. Si se considera el estudio de Ramos y Prideaux (2014), realizado en 
Palenque (México), se observa cómo el mayor beneficio es para los capitales extranjeros. Por último, se propone es investigar las patologías que causa el turismo, o que se generan como consecuencia del turismo, como la prostitución, estudiada por Naser (2013) en Egipto.

El tópico que aborda temas de gestión cultural y cuenta con cuatro ítems, respecto a los que ya se había llegado al más alto nivel de consenso en la primera ronda, ratificado en la segunda. No obstante, es en este tópico en el que se produce el único caso de línea de investigación propuesta con la que los expertos se han mostrado "totalmente en desacuerdo". Esta línea proponía estudiar el grado de conocimiento que los interesados del turismo tienen acerca del patrimonio, pero ya, en una de las preguntas de encuadre, opinaban que se cuenta con un conocimiento muy limitado de los valores de excepcionalidad de la UNESCO, por lo que no es necesario investigar este hecho.

Las tres líneas restantes que se han consensuado en el tópico de gestión cultural son las siguientes. La discriminación de los recursos culturales puestos en valor por el turismo, dado que, en la mayoría de los SPM, existe un reduccionismo importante, al que ya hacían referencia Kaltenborn et al. (2013), lo que conlleva a que la visita sea muy condicionada y, a la vez, muy corta. La siguiente línea de investigación es la de conocer las estrategias de interpretación patrimonial, éstas de cara al visitante y a los diferentes segmentos de visitantes, es decir, se debe de investigar cuál es la mejor forma de poner en valor turístico el patrimonio para niños, para adultos, para extranjeros, etc. Esta línea ya ha sido observada en trabajos como el de Fuentes y Gallardo (2014). La tercera línea de investigación se refiere a abordar por separado el patrimonio cultural material e inmaterial. Para esta línea, los investigadores participantes comentaron que el patrimonio inmaterial es poco estudiado, pero que es el que le otorga un simbolismo importante al patrimonio material, como describen Zanirato y Tomazzoni (2015), según los cuales, cuando los valores patrimoniales se convierten en objetos de consumo, no se relaciona con la valoración de las identidades locales, lo que lleva a tener importantes riesgos de perder la identidad cultural.

En el último tópico, relacionado con los impactos económicos, el consenso es claro desde la primera ronda y confirmado en la segunda, en la que se establece la importancia de cuatro líneas de investigación, que son, en primer lugar, verificar los impactos económicos generados por la actividad turística en la sociedad local, desde los positivos, que son los que principalmente se establecen por medio del empleo, hasta los negativos, que conlleva el terciarizar un determinado territorio, como la especulación y la marginación de residentes en espacios turísticos (Viu et al., 2008). En segundo lugar, investigar los beneficios de la actividad turística a escala municipal, partiendo de los que produce al propio municipio mediante impuestos locales, dado que los beneficios a la oferta de carácter comercial suelen ser más evidentes.

En tercer lugar, evaluar la inversión pública y privada en turismo en los SPM; es muy frecuente observar una descoordinación entre las autoridades municipales y los empresarios interesados en turismo. Así, se debe investigar cuál ha sido la inversión privada, ya que es más factible que se adapte a los cambios de la demanda, por lo que la administración municipal deberá complementar estos esfuerzos y encabezar otros basados en la gobernanza, como señalan Ryan et al. (2011). En cuarto lugar, analizar la caracterización y evolución del empleo generado por la actividad turística. Esta línea no solo debe contemplar el total de empleos generados, que, como afirman Ramos y Pideraux (2014), en ocasiones, no son los mejores para la localidad, sino que se debe investigar su calidad. 
En el tercer grupo de resultados se recogen las líneas de investigación que fueron propuestas por los expertos y consensuadas en la segunda ronda del Delphi para los siguientes tópicos:

\section{Gestión especifica de turismo}

Investigar sobre las redes que se establecen en las diferentes instancias de gestión provocadas por la propia transversalidad del turismo, debido a que es frecuente observar un grado importante de descoordinación en las acciones turísticas, en las que no se optimizan los recursos, tanto financieros como humanos (Paül, 2013), para lo que, incluso, se ha propuesto la creación de comisiones intergubernamentales.

Investigar la seguridad que deberán ofrecer los destinos turísticos. Actualmente, existen SPM que atraviesan crisis de seguridad por diversos motivos y es importante que las políticas públicas se orienten en este sentido. Como comenta Korstanje (2014), el propio viaje ya implica una cierta inseguridad.

\section{Flujos turísticos}

Investigar acerca de las posibilidades de la aplicación de las nuevas tecnologías para realizar el seguimiento de los visitantes en los SPM. Es importante dar seguimiento a los viajeros, como comentan Donaire et al. (2015), superando las inconveniencias de hacer seguimiento con la técnica de observación directa y emprender nuevos modelos de seguimiento, como el uso del GPS.

Investigar acerca de las rutas turísticas más utilizadas. La intención de esta línea es observar cuál es la distribución de los visitantes en los SPM y, con ello, evitar altas concentraciones que pueden llegar a afectar a la experiencia de los visitantes y, al mismo tiempo, dar uso a otras zonas de los SPM que aún no han sido potenciadas y que pueden coadyuvar al desarrollo económico de la localidad.

\section{Marketing}

Investigar productos y marcas a nivel destino, es decir, además de potenciar la marca UNESCO, identificar alternativas de posicionamiento de marcas locales, con productos endógenos capaces de producir beneficios locales.

Investigar el impacto de la promoción turística, los expertos proponentes hacen referencia a que debe observarse la promoción internacional, porque se debate si los SPM son capaces de atraer turismo internacional al destino.

\section{Impactos sociales}

Una línea relacionada con aspectos de participación social, el asociacionismo y sensibilización de la población infantil en materia de turismo, en la que se debe buscar una mayor participación de la sociedad de manera organizada, incluso pudiendo constituirse como organizaciones no gubernamentales en defensa del patrimonio. 


\section{CONCLUSIONES}

De las preguntas de encuadre, se obtiene que la tendencia es seguir investigando estudios de caso, quizá porque en cada SPM la inserción del turismo, en cada territorio, cuenta con sus propias características y no siempre se pueden replicar las mismas metodologías. No obstante, se pueden armonizar y aplicar en destinos que compartan el mismo grado de desarrollo. Es importante seguir estudiando la relación turismo-patrimonio en los SPM de forma general, dado que el corpus normativo de la UNESCO es de aplicabilidad general.

La investigación de los SPM y el turismo se vislumbra compleja. Entre otras cuestiones, los expertos opinan que el turismo no cumple con todos los principios internacionales de sostenibilidad. El consenso va en relación a que la actividad turística en muy pocas ocasiones puede contribuir a la preservación del patrimonio, lo que lleva a pensar, nuevamente, en la importancia de investigar este fenómeno que cada día crece y, por tanto, representa una buena o mala oportunidad para el desarrollo en los SPM.

En la primera pregunta del estudio Delphi, los expertos llegan al consenso de que el reconocimiento como SPM sí puede incrementar flujos de visitantes, siendo necesario establecer la capacidad de acogida para que se intente practicar acciones sostenibles, de entre las que se destaca adaptar productos turísticos patrimoniales en torno a diversos segmentos de mercado, además del puramente cultural, y que éstos deben ser atractivos, en principio, para sus propios habitantes

Es notorio que, según el panel de expertos, los involucrados en el turismo de los SPM no conocen los valores por los que las ciudades fueron reconocidas, lo que plantea una nueva cuestión, relacionada con la importancia del reconocimiento de excepcionalidad con el que cuentan los SPM, porque abre una línea de investigación acerca de la oferta cultural actual y su apego a esta reconocida excepcionalidad, es decir, el patrimonio está siendo eclipsado por otros valores que resultan atractivos al visitante.

De forma general, este artículo destaca las líneas de investigación que pueden ser abordadas en relación a los impactos generados por el turismo en los SPM, líneas que en ningún momento son limitativas, y son el resultado de un análisis previo de la literatura registrada en Scopus y consensuada por grandes expertos del tema, del panorama mundial. Asimismo, en ningún caso se pretende que todas sean abordadas desde una misma disciplina, siendo ésta una oportunidad que ofrece el estudio del turismo, ya que es necesaria la intervención de una gran cantidad de ciencias y disciplinas que aborden el tema de manera científica y multidisciplinar, reconociendo que la geografía humana logra recoger gran parte de las cuestiones abordadas.

De forma general, tras el estudio Delphi, se obtiene que las líneas de investigación altamente consensuadas son un total treinta y seis. Los tópicos que ofrecen una mayor cantidad de líneas de investigación, en orden descendente, son el marketing y los flujos turísticos, con seis líneas respectivamente. Los tópicos de gestión, análisis territorial e impactos sociales, con cinco líneas de investigación cada uno de ellos. El tópico de impactos sociales se mantiene con cuatro líneas de investigación. La gestión cultural con tres líneas, siendo uno de los tópicos en el que, por consenso, se desechó una línea propuesta. En relación a la sostenibilidad, se puede concluir que ésta se debe abordar de forma transversal, este es el tópico en el que se encontraron más publicaciones en la revisión de la literatura y, por tanto, genera menos nuevas líneas de investigación. En la Tabla 5, se recogen las líneas prioritarias a investigar en la compleja relación turismo-SPM, identificadas por consenso de los expertos participantes en esta investigación. 


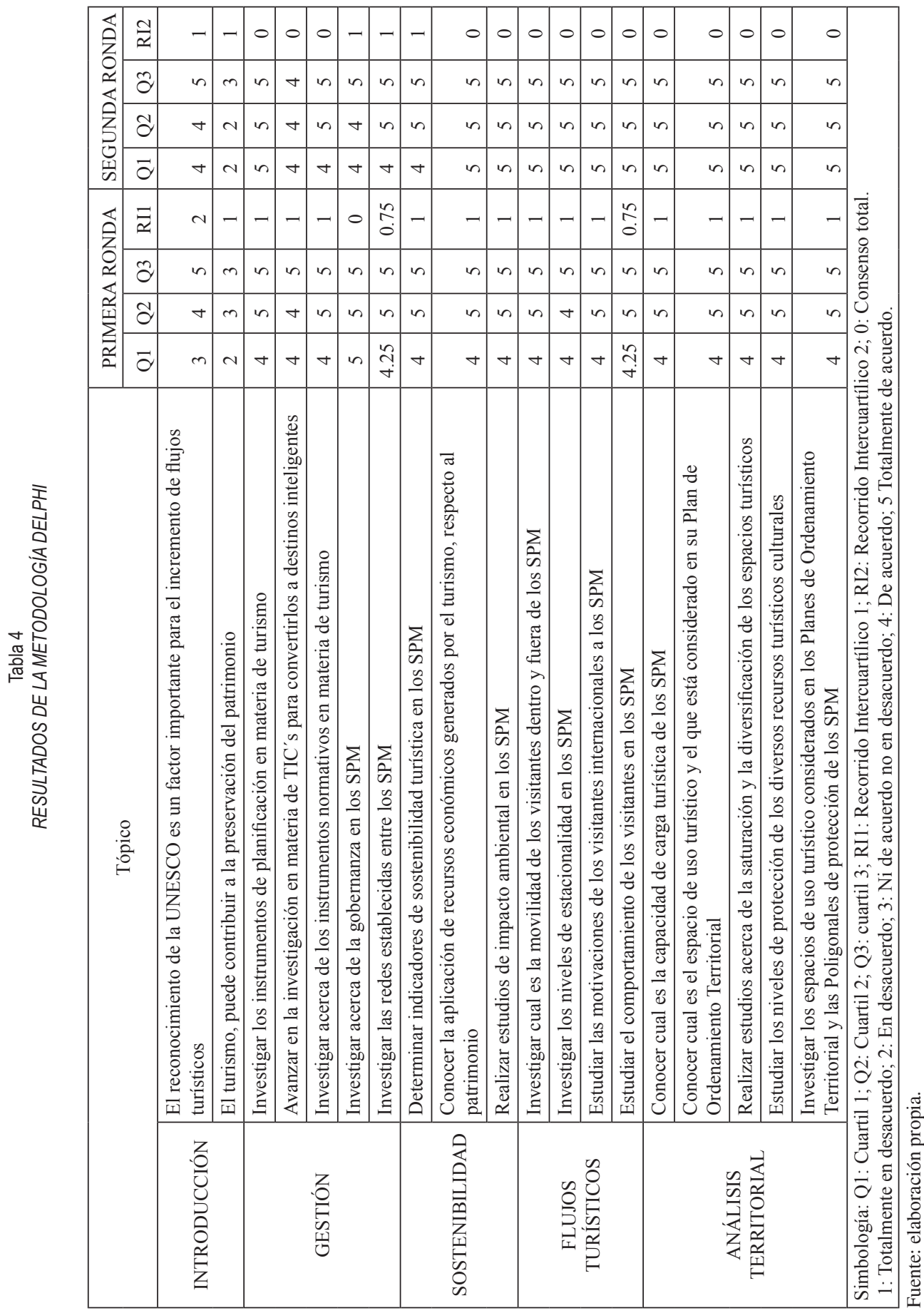




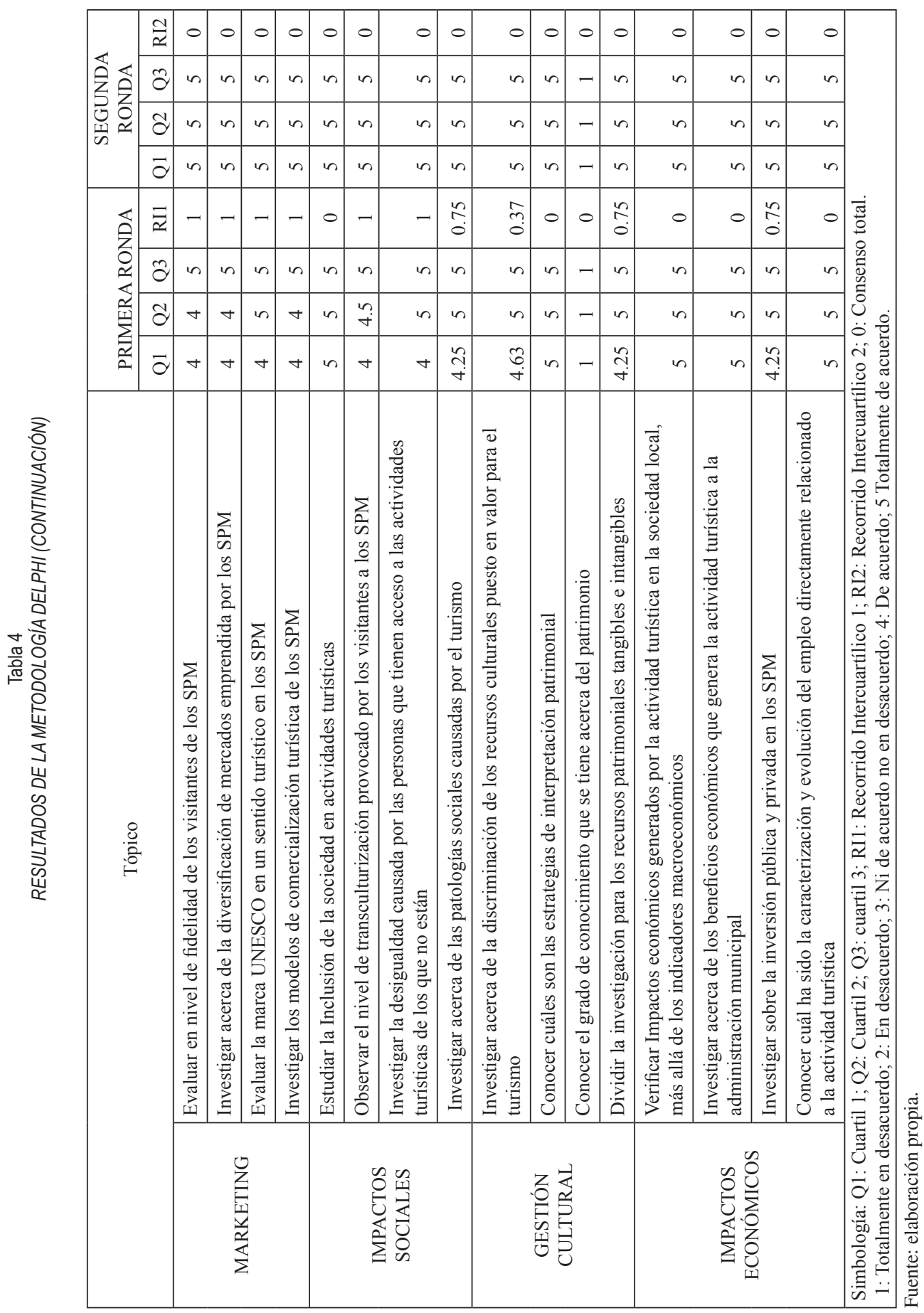


Tabla 5

RESULTADOS DEL ESTUDIO DELPHI

\begin{tabular}{|c|c|c|c|}
\hline TÓPICO & LÍNEAS DE INVESTIGACIÓN & TÓPICO & LÍNEAS DE INVESTIGACIÓN \\
\hline \multirow{4}{*}{ GESTIÓN } & Instrumentos de planificación en materia de turismo & \multirow{6}{*}{ MARKETING } & Nivel de fidelidad de los visitantes de los SPM \\
\hline & Nuevas TIC's para convertirlos a destinos inteligentes & & La diversificación de mercados emprendida por los SPM \\
\hline & Instrumentos normativos en materia de turismo & & $\begin{array}{l}\text { Evaluar la marca UNESCO en un sentido turistico en } \\
\text { Los SPM }\end{array}$ \\
\hline & $\begin{array}{l}\text { Las redes multisectoriales, provocadas por la } \\
\text { transversalidad del turismo }\end{array}$ & & Los modelos de comercialización turística de los SPM \\
\hline \multirow{2}{*}{ SOSTENIBILIDAD } & $\begin{array}{l}\text { La aplicación de recursos económicos generados por } \\
\text { el turismo, respecto al patrimonio }\end{array}$ & & Productos y marcas a nivel destino \\
\hline & Estudios de impacto ambiental en los SPM & & Lugares donde se realiza la promoción del destino \\
\hline \multirow{6}{*}{$\begin{array}{l}\text { FLUJOS } \\
\text { TURÍSTICOS }\end{array}$} & La movilidad dentro y fuera de los SPM & \multirow{4}{*}{$\begin{array}{l}\text { IMPACTOS } \\
\text { SOCIALES }\end{array}$} & $\begin{array}{l}\text { Estudiar la inclusión de la sociedad en actividades } \\
\text { turisticas }\end{array}$ \\
\hline & Los niveles de estacionalidad en los SPM & & $\begin{array}{l}\text { El nivel de transculturización provocado por los } \\
\text { visitantes a los SPM }\end{array}$ \\
\hline & $\begin{array}{l}\text { Las motivaciones de los visitantes internacionales a } \\
\text { los SPM }\end{array}$ & & Las patologias sociales causadas por el turismo \\
\hline & El comportamiento de los visitantes en los SPM & & $\begin{array}{l}\text { Aspectos de participación social, asociacionismo y } \\
\text { sensibilización de la población infantil }\end{array}$ \\
\hline & $\begin{array}{l}\text { Seguimiento de visitantes con inclusión de nuevas } \\
\text { tecnologias }\end{array}$ & \multirow{3}{*}{$\begin{array}{l}\text { GESTIÓN } \\
\text { CULTURAL }\end{array}$} & $\begin{array}{l}\text { La discriminación de los recursos culturales, puestos en } \\
\text { valor para el turismo }\end{array}$ \\
\hline & El estudio de las rutas turísticas mas utilizadas & & Las estrategias de interpretación patrimonial \\
\hline \multirow{5}{*}{$\begin{array}{l}\text { ANÁLISIS } \\
\text { TERRITORIAL }\end{array}$} & La capacidad de acogida turistica de los SPM & & $\begin{array}{l}\text { Investigar distinguiendo el patrimonio tangible e } \\
\text { intangible }\end{array}$ \\
\hline & $\begin{array}{l}\text { El espacio de uso turistico y el que está considerado } \\
\text { en su Plan de Ordenamiento Territorial }\end{array}$ & \multirow{4}{*}{$\begin{array}{l}\text { IMPACTOS } \\
\text { ECONÓMICOS }\end{array}$} & $\begin{array}{l}\text { Verificar los impactos económicos generados por la } \\
\text { actividad turistica en la sociedad, mas allá de los } \\
\text { indicadores macroeconómicos }\end{array}$ \\
\hline & $\begin{array}{l}\text { Estudios acerca de la saturación y la diversificación } \\
\text { de los espacios turísticos }\end{array}$ & & La recaudación municipal por conceptos de turismo \\
\hline & $\begin{array}{l}\text { Niveles de protección de los diversos recursos } \\
\text { turisticos culturales }\end{array}$ & & La inversión de la oferta turistica en los SPM \\
\hline & $\begin{array}{l}\text { Espacios y uso turístico en relación a las poligonales } \\
\text { de protección }\end{array}$ & & $\begin{array}{l}\text { La caracterización y evolución del empleo directamente } \\
\text { relacionado a la actividad turistica }\end{array}$ \\
\hline
\end{tabular}

Fuente: elaboración propia.

\section{BIBLIOGRAFÍA}

AAS, C., LADKIN, A. y FLETCHER, J. (2005): «Stakeholder collaboration and heritage management». Annals of Tourism Research, 32, 28-48.

BATISSE, M. (1992): «The struggle to save our world heritage» en Environment, 34, 12-20.

BECK, W. (2006): «Narratives of world heritage in travel guidebooks». International Journal of Heritage Studies, 12 6, 521-535.

BEESON, S. y DOGANER, S. (2013): «Visual assessment of San Antonio Franciscan Mission churches in San Antonio for sustainable cultural heritage tourism». WIT Transactions on the Built Environment, vol. 131, 403-415.

BELL, V. (2013): «The politics of managing a World Heritage Site the complex case of Hadrian $>$ S Wall»: Leisure Studies, Vol. 32 (2), 115-132.

BERLINER, D. (2012): «Multiple nostalgias The fabric of heritage in Luang Prabang Lao PDR». Journal of the Royal Anthropological Institute, vol. 18 (4), 769-786.

BERNIER, X. y SUTTON, K. (2012): «Les fragmentations d'un territoire fortifié Briançon à l'épreuve de sa labellisation» en Mappemonde, 108 4,1-21.

BROWN, P. y KING, B. (2013): «UNESCO and the strategic assessment what it means for ports and shipping», en Coasts and Ports 2013, 151-156.

BRUMANN, C. (2014): «Heritage agnosticism a third path for the study of cultural heritage». Social Anthropology, vol. 22 (2), 173-188. 
ČAUŠEVIĆ, S. y TOMLJENOVIĆ, R. (2003): «World Heritage Site, tourism and city>S rejuvenation the case of Poreč, Croatia». Tourism, vol. 51 (4), 417-426.

CHENG, M., WONG, I. y LIU, M. (2013): «A cross-cultural comparison of world heritage site image the case of hue». Tourism Analysis, vol.18 (6), 707-712.

COBURN, B. (1984): «Sagarmatha managing a Himalayan World Heritage Site». Parks, vol. 9 (2), 10-19.

CÓRDOBA, J. (2009): «Turismo, desarrollo y disneyzación ¿Una cuestión de recursos o de ingenio?». Investigaciones Geográficas, $\mathrm{n}^{\circ}$ 70, 33-54.

DAENGBUPPHA, J., HEMMINGTON, N. y WILKES, K. (2006): «Using grounded theory to model visitor experiences at heritage sites Methodological and practical issues». Qualitative Market Research, vol. 9 (4), 367-388.

DAVIS, D. y WEILER, B. (1992): «Kakadu National Park conflicts in a world heritage area». Tourism Management, vol. 13 (3), 313-320.

DE LA CALLE, M. y GARCÍA, M. (2015): «Turismo en el mundo maya. Flujos de visitantes en el Sitio Arqueológico de Copán». Cuadernos de Turismo, no 36, 101-127.

DONG, X., ZHANG, J., ZHI, R., ZHONG, S. y LI, M. (2011): «Review on the websites and virtual tourism of World Heritage sites in China», en 2nd International Conference on Information Science and Engineering, Actas del Segundo Congreso Internacional de Informacion, Ciencias e Ingenieria, ICISE 2010 ART 5691830, 6112

DONAIRE, J., GALI, N. y ROYO-VELA, M. (2015): «El uso del GPS para el análisis del comportamiento espacio-temporal de los turistas. Pre-test en el Valle de Boí». Cuadernos de Turismo, $\mathrm{n}^{\mathrm{o}} 35,117-131$.

DONOHOE, H. M. y NEEDHAM, R.D. (2009): «Moving best practice forward: delphi. Characteristics, advantages, potential problems and solutions». International Journal of Tourism Research, vol. 11 (5), 415-437.

DUVAL, M. y GAUCHON, C. (2007): «Analyse critique d`une politique d`aménagement du territoire, les Opérations Grands Sites». Annales de Geographie, vol. 116, nº 654, 147-168.

FATIN, H., AMIRAH, A. y KHAIRANI, N. (2014): «Determinants of tourist perception towards responsible tourism A study at Malacca world heritage site» en Theory and Practice in Hospitality and Tourism Research Proceedings of the 2nd International Hospitality and Tourism Conference 2014, 269-273.

FERNÁNDEZ, P. (2007): «K〉Gari or paradise island» en Proceedings of the 8th International Conference on the Mediterranean Coastal Environment, MEDCOAST 2007, 1, 235-246.

FUENTES, F. y GALLARDO, L. (2014): «An aproach to the key success factors for heritage sites management». TMStudies, vol. 10, 186-192.

GARCÍA, M., DE LA CALLE, M. y MINGUEZ, M.C. (2011): «Capacidad de carga turística y espacios patrimoniales. Aproximación a la estimación de la capacidad de carga del conjunto arqueológico de Carmona (Sevilla, España)». Boletín de la Asociación de Geógrafos Españoles, n $57,219-241$.

GARCÍA, M. y DE LA CALLE, M. (2012): «Capacidad de carga en grandes recursos turístico-culturales». Anales de Geografia de la Universidad Complutense de Madrid, n 32 (2), 253-274. 
GARROD, B. y FYALL, A. (2000): «Managing heritage tourism». Annals of Tourism Research, vol. 27 (3), 682-708.

GLEMŽA, J. (2007): «Heritage and cultural tourism in Vilnius», en Urban Heritage Research, Interpretation, Education Scientific Conference, Proceedings, 98-100.

HARDIMAN, N. y BURGIN, S. (2013): «World Heritage Area listing of the Greater Blue Mountains Did it make a difference to visitation?». Tourism Management Perspectives, vol. 6, 63-64.

HAWKINS, D., CHANG, B. y WARNES, K. (2009): «A comparison of the National Geographic Stewardship Scorecard Ratings by experts and stakeholders for selected World Heritage destinations» . Journal of Sustainable Tourism, vol. 17 (1), 71-90.

HAWES, M. (1996): «A walking track management strategy for the Tasmanian Wilderness World Heritage Area». Australian Parks and Recreation, vo. 32 (2), 18-23.

HENNESY, E. y MCCLEARY, A. (2011): «Nature»s Eden? the production and effects of 〈pristine〉 nature in the Galápagos Islands» en Island Studies Journal, 6 2, 131-156.

HILL, K. y FOWLES, J. (1975): «The methodological worth of the Delphi forecasting technique». Technological Forecasting and Social Change, vol.7 (2), 179-192.

JEON, M., KANG, M. y DESMARAIS, E. (2016): «Residents Perceived Quality Of Life in a Cultural-Heritage Tourism Destination». Applied Research in Quality of Life, vol. 11 (1), 105-123.

JIMURA, T. (2011): «The impact of world heritage site designation on local communities A case study of Ogimachi, Shirakawa-mura, Japan». Tourism Management, vol. 32 (2), 288-296.

JURENIENE, V. y RADZEVICIUS, M. (2014): «Models of cultural heritage management». Transformations in Business and Economics, vol. 13 (2), 236-256.

KALTENBORN, B., THOMASSEN, J., WOLD, L., LINNELL, J. y SKAR, B. (2013): «World Heritage status as a foundation for building local futures? A case study from Vega in Central Norway». Journal of Sustainable Tourism, vol. 21 (1), 99-116.

KAVOURA, A. y BITSANI, E. (2013): «Managing the World Heritage Site of the Acropolis, Greece». International Journal of Culture, Tourism, and Hospitality Research, vol. 7 (1), 58-67.

KIM, S., WONG, K. y CHO, M. (2007): «Assessing the economic value of a world heritage site and willingness-to-pay determinants A case of Changdeok Palace». Tourism Management, vol. 28 (1), 317-322.

KIRKPATRICK, J. (2001): «Ecotourism, local and indigenous people, and the conservation of the Tasmanian Wilderness World Heritage Area». Journal of the Royal Society of New Zealand, vol. 31 (4), 819-829.

KOLLWITZ, H. (2011): «Evaluating cruise demand forecasting practices: A Delphi approach». Royal Anthropological Institute, vol. 18 (4), 769-786.

KORSTANJE, M. (2014): «De la fobia al miedo de viajar: explorando la psicología profunda del turista». Papers de Turisme, $\mathrm{n}^{\circ}$ 56, 101-118.

LARSON, L. y POUDYAL, N. (2012): «Developing sustainable tourism through adaptive resource management. A case study of Machu Picchu, Peru». Journal of Sustainable Tourism, vol. 20 (7), 917-938. 
LAZZAROTTI, O. (2000): «Patrimoine et tourisme. Un couple de la mondialisation». Mappemonde, vol. 57 (1), 12-16.

LI, S., HU, X., TANG, Y., HUANG, C. y XIAO, W. (2014): «Changes in lacustrine environment due to anthropogenic activities over 240 years in Jiuzhaigou National Nature Reserve, southwest China». Quaternary International, no 349, 367-375.

LOZANO, S. y GUTIÉRREZ, E. (2011): «Efficiency analysis of EU-25 member states as tourist destinations» en International Journal of Services, Technology and Management, 15 1-2, 69-88.

MÍNGUEZ, C. (2013): «La gestión de la oferta turístico-cultural en grandes hitos patrimoniales. El caso de Patrimonio Nacional». Boletín de la Asociación de Geógrafos Españoles, 63, 223-246.

MOURIÑO, H. (2013): «Ordinal regression models to describe tourist satisfaction with Sintra〉s world heritage», en AIP Conference Proceedings, 1558, pp. 1.885-1.888.

NÄSER, C. (2013): «Landscapes of Desire Tourists, Touts and Sexual Encounters at the World Heritage Site of Thebes». Archaeologies, vol. 9 (3), 398-426.

OKECH, R. (2010): «Socio-cultural impacts of tourism on world heritage sites Communities perspective of lamu kenya and Zanzibar Islands». Asia Pacific Journal of Tourism Research, vol. 15 (3), 339-351.

PALAU, R., FORGAS, S., SÁNCHEZ, J. y PRATS, L. (2013): «Tourist Behavior Intentions and the Moderator Effect of Knowledge of UNESCO World Heritage Sites The Case of La Sagrada Familia». Journal of Travel Research, vol. 52 (3), 364-376.

PARLETT, G., FLETCHER, J., y COOPER, C. (1995): «The impact of tourism on the Old Town of Edinburgh». Tourism Management, vol. 16 (5), 355-360.

PATUELLI, R., MUSSONI, M. y CANDELA, G. (2013): «The effects of World Heritage Sites on domestic tourism A spatial interaction model for Italy». Journal of Geographical Systems, vol. 15 (3), 369-402.

PAÜL, A. (2013): «Las políticas culturales y sus repercusiones en la ciudad». Scripta Nova. vol. 17, 425-462.

PÉREZ Y. y NEL, L. (2013): «Propuesta de indicadores para evaluar la sostenibilidad de la actividad turística. El caso del Valle de Viñales Cuba», Anales de Geografía de la Universidad Complutense, $\mathrm{n}^{\circ} 33$ (1), 193-210.

PIÉDROLA, I., ARTACHO, C. y VILLASECA M. (2012): «Tourism and learn Spanish in historic cities A case study in Córdoba». Studies in Fuzziness and Soft Computing, $\mathrm{n}^{\circ}$ 287, 305-318.

POKHAREL, S. y MUTHA, A. (2009): «Perspectives in reverse logistics: A review». Ressources, Conservation and Recycling, vol. 53 (4), 175-182.

POLANCO, V. (2009): «Patrimonio urbano y turismo cultural en la ciudad de México Las chinampas de Xochimilco y el centro histórico». Andamios, vol. 6 (12), 69-94.

POPP, H. (2001): «Weltkulturerbe aït Ben Haddou Morokko. Lokale vermarktung eines standorts von globalem interesse». Geographische Rundschau, vol. 53 (6), 44-49.

PORIA, Y., REICHEL, A. y COHEN, R. (2013): «Tourists perceptions of World Heritage Site and its designation». Tourism Management, vol. 35, 272-274.

PULIDO-FERNANDEZ, J.I. (2007): «El turismo en el desarrollo económico de los parques naturales andaluces. Un análisis Delphi de los principales déficit». Revista de Estudios Empresariales, $\mathrm{n}^{\mathrm{o}}$ 1, 110-137. 
RAMOS, A. y PRIDEAUX, B. (2014): «Indigenous ecotourism in the Mayan rainforest of Palenque empowerment issues in sustainable development». Journal of Sustainable Tourism, vol. 22 (3), 461-479.

RODRIGUEZ, I. y PULIDO-FERNANDEZ, J.I. (2010): «Factores condicionantes de la sostenibilidad como una dimensión estratégica del desarrollo turístico mexicano», Cuadernos de Turismo, $\mathrm{n}^{\circ}$ 25, 125-146.

ROQUE, H., HENRIQUES, M. y MOURIÑO, H. (2012): «How do tourists look at Sintra>S world heritage? ». WIT Transactions on Ecology and the Environment, vol. 161, 369-380.

RUIZ, A. y PULIDO, J.I. (2015): «El impacto del turismo en los Sitios Patrimonio de la Humanidad. Una revisión de las publicaciones científicas de la base de datos Scopus». Pasos. Revista de Turismo y Patrimonio Cultural, vol. 13 (5), 1.247-1.264.

RYAN, C., CHAOZHI, Z. y ZENG, D. (2011): «The impacts of tourism at a UNESCO heritage site in China a need for a meta-narrative? The case of the Kaiping diaolou». Journal of Sustainable Tourism, vol. 19 (6), 747-765.

SCHMITT, T. (2005): «Jemaa el Fna Square in Marrakech Changes to a social space and to a UNESCO masterpiece of the oral and intangible heritage of humanity as a result of global influences». Arab World Geographer, vol. 8 (4), 173-195.

SHAFER, C. y INGLIS G. (2000): «Influence of social, biophysical, and managerial conditions on tourism experiences within the Great Barrier Reef World Heritage Area». Environmental Management, vol. 26 (1), 73-87.

SHEN, S., GUO, J. y WU, Y. (2014): «Investigating the Structural Relationships among Authenticity, Loyalty, Involvement, and Attitude toward World Cultural Heritage Sites An Empirical Study of Nanjing Xiaoling Tomb, China». Asia Pacific Journal of Tourism Research, vol. 19 (1), 103-121.

SICHENZE, A; MACAIONE, I. Y INSETTI, M. (2006): «The discovery of the nature-city and the re-generative strategies» en WIT Transactions on Ecology and the Environment, 97, 95-104.

SU, Y. y LIN, H. (2014): «Analysis of international tourist arrivals worldwide The role of world heritage sites». Tourism Management, vol. 40, 46-58.

SU, M. y WALL, G. (2014): «Community participation in tourism at a world heritage site Mutianyu great wall, Beijing, China». International Journal of Tourism Research, vol. 16 (2), 146-156.

SU, X. y TEO, P. (2008): «Tourism politics in Lijiang, China An analysis of State and local interactions in tourism development». Tourism Geographies, vol. 10 (2), 150-168.

SUN, J. (2014): «The integration and development of heritage resources based on RMP analysis a case study of Songshan world Geopark». Advanced Materials Research, vol. 889-890, 1.653-1.659.

TUCKER, H. y CARNEGIE, E. (2014): «World heritage and the contradictions of "universal value"». Annals of Tourism Research, vol. 47, 63-76.

TODT, H., HERDER, D. y DABIJA, D. (2008): «The role of monument protection for tourism». Amfiteatru Economic, vol. 10, 292-297.

VERA, S. y SONG, H. (2014): «A review of Delphi forecasting research in tourism». Current Issues in Tourism, vol. 18 (12), pp. 1.099-1.131. 
VIU, J., FERNÁNDEZ, J. y CARALT, J. (2008): «The impact of heritage tourism on an urban economy The case of Granada and the Alhambra».Tourism Economics, vol. 14 (2), 361-376.

WAGER, J. (1995): «Developing a strategy for the Angkor World Heritage Site». Tourism Management, vol. 16 (7), 515-523.

WALLACE, G. (1993): «Visitor management lessons from Galapagos National Park», en Ecotourism a guide for planners and managers, vol. 1, 55-81.

WANG, T. y ZAN, L. (2011): «Management and presentation of Chinese sites for UNESCO World Heritage List UWHL». Facilities, vol. 29 (7), 313-325.

WEBSTER, J. y WATSON, R. (2002): «Analyzing the past to prepare for the future Writing a literature review». Management Information Systems Quarterly, vol. 262, 3.

WINTER, T. (2007): «Rethinking tourism in Asia». Annals of Tourism Research, vol. 34 (1), 27-44.

WU, M., WALL, G. y ZHOU, L. (2014): «A free pricing strategy at a major tourist attraction The Case of West Lake, China». Journal of Destination Marketing and Management, vol. 3 (2), 96-104.

XU, H. y DAI, S. (2012): "A system dynamics approach to explore sustainable policies for Xidi, the world heritage village». Current Issues in Tourism, vol. 15 (5), 441-459.

YAMAMURA, T., ZHANG, T. y FUJIKI, Y. (2006): «The social and cultural impact of tourism development on world heritage sites A case of the Old Town of Lijiang, China, 2000-2004». WIT Transactions on Ecology and the Environment, vol. 97, 117-126.

YANG, L. y WALL, G. (2014): «Planning for Ethnic Tourism» Ed. Ashgate Publishing Limited, England, 293 pp.

YUKSEL, F., BRAMWELL, B. y YUKSEL, A. (1999): «Stakeholder interviews and tourism planning at Pamukkale, Turkey» . Tourism Management, vol. 20 (3), 351-360.

ZHANG, C., FYALL, A. y ZHENG, Y. (2015): «Heritage and tourism conflict within world heritage sites in China a longitudinal study». Current Issues in Tourism, vol. 18 (2), 110 136.

ZHU, Y. (2012): «When the Global Meets the Local in Tourism-Cultural Performances in Lijiang as Case Studies». Journal of China Tourism Research, vol. 8 (3), 302-319.

ZANIRATO, H. y TOMAZZONI, L. (2015): «Patrimonio, turismo y trasfiguraciones en las relaciones identidarias El Pelouriho (Salvador - Bahía) y Porto Rico (Paraná), Brasil». Estudios y Perspectivas en Turismo, nº 24 (2), 222-243. 
